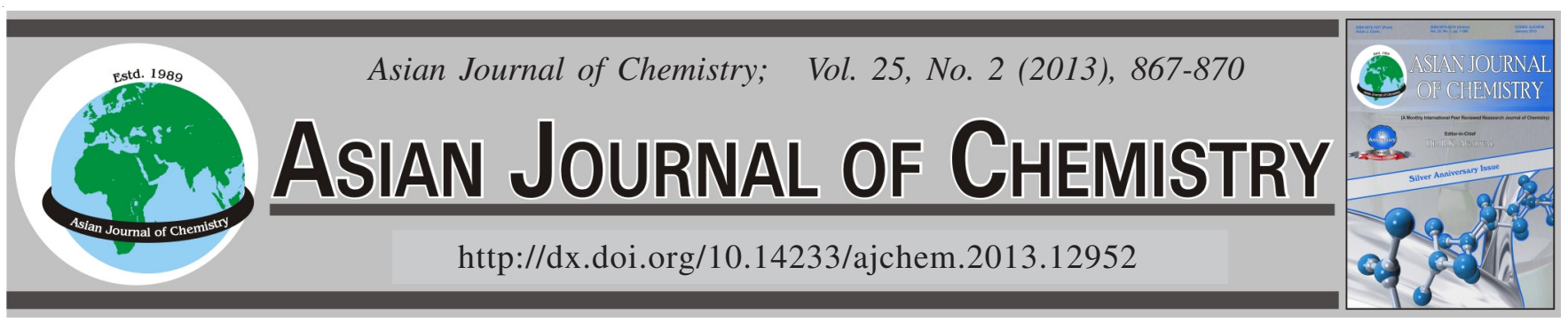

\title{
Experimental Investigation of the Interaction Between Human Serum and Troxerutin by Fluorescence Spectroscopy
}

JiHUA ZHANG, ZHIMIN ZHAO and TIANHU WANG

College of Science, Nanjing University of Aeronautics and Astronautics, Nanjing 210016, P.R. China

*Corresponding author: Fax: +86 25 84892011; Tel: +86 25 84892011; E-mail: nuaazhzhm@126.com

Based on fluorescence spectroscopy technology, the interaction between human serum and troxerutin is investigated. The experimental results showed that only a fluorescence peak at $330 \mathrm{~nm}$ arises when the human surem is excited at $250-290 \mathrm{~nm}$. When troxerutin is added, the position of fluorescence peaks has a slight blue shift and its intensity is weakened. It can be obtained that $290 \mathrm{~nm}$ is the most suitable excited wavelength for the research of serum-troxerutin's interaction. Moreover, Gaussion fitting curves reveal that the fluorescence of human serum is caused by two fluorophores. The values of $\mathrm{I}_{325}$ and $\mathrm{I}_{350}$ decrease after the addition of troxerutin, which illustrates that both fluorophores can interact with troxerutin.

Key Words: Human serum, Troxerutin, Fluorescence spectroscopy, Optimal excitation wavelength, Gaussion fitting.

\section{INTRODUCTION}

Fluorescence is generally defined as a luminescence emission that is caused by the flow of some form of energy into the emitting body, which is named fluorophore. Scilicet only fluorophores can emit fluorescence when excited by light ${ }^{1}$. Fluorescence spectroscopy is an important method to study the interaction of substances with protein, which can provide some information about structures of chemical compound $^{2,3}$ or changes of the environment of fluorophores ${ }^{4,5}$.

Human serum contains 6-8 \% solids, including macromolecules such as albumin, antibodies and other globulins and enzymes; peptide and lipid-based hormones and cytokines; as well as certain nutritive organic materials in small amounts, such as amino acids, glucose and fats ${ }^{1}$. Human serum albumin (HSA) is the most abundant protein in human serum. It constitutes about half of the blood serum protein and most drug. The structure of human serum albumin is in Fig. 1. Human serum albumin plays an important role in the transport and disposition of endogenous and exogenous ligands present in blood $^{6}$. More than $90 \%$ of the medicine that is taken bound to serum albumin. Therefore, the interaction between drugs and serum albumin has been attracting increasing attention with the development of fluorescence spectroscopy technology. Based on fluorescence spectroscopy, fluorescence quenching mechanism of drugs and albumin can be investigated and some parameter, binding constant and number of binding sites, can be obtained $d^{7-10}$. These studies demonstrated the power of

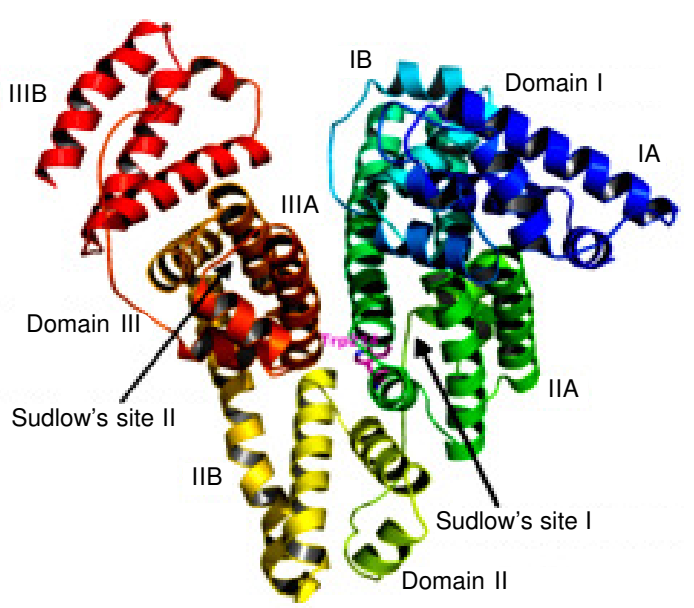

Fig. 1. Structure of human serum albumin

fluorescence spectroscopy technology in pharmacological research.

Troxerutin (Fig. 2) shows a marked affinity for the venous wall. The highest uptake in the outer wall region is likely to result from transport through the vasa vasorum due to the rheologic properties of the drug ${ }^{11}$. We reported the binding of troxerutin to bovine serum albumin ${ }^{7}$. The purpose of this study is to investigate the behaviour of the interaction between human serum and troxerutin for expounding pharmacological actions and determine the optimal excitation wavelength for serum-troxerutin interaction. 


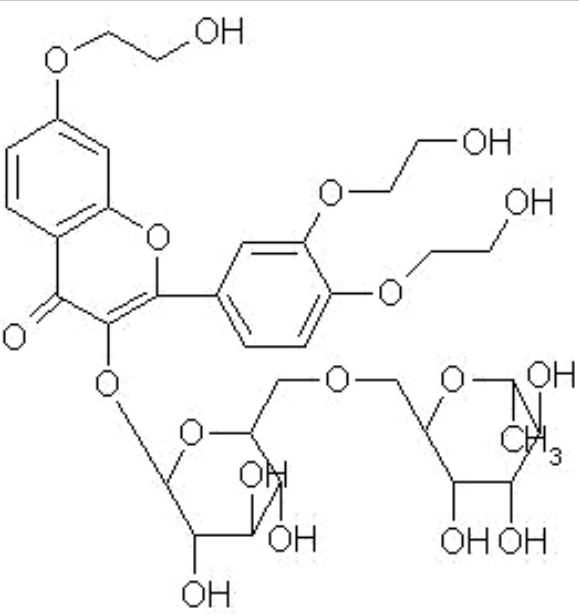

Fig. 2. Structure of troxerutin

\section{EXPERIMENTAL}

The human serum sample was provided by the hospital of NUAA. The troxerutin sample was obtained from Shanxi Yabao Pharmaceutical Group Co. Ltd. (Shanxi, China). The human serum stock solution $\left(1.0 \mu \mathrm{M} \mathrm{L}^{-1}\right)$ was prepared in phosphate buffer solution ( $\mathrm{pH} 7.4$ ) containing $0.1 \mathrm{~mol} \mathrm{~L}^{-1}$ $\mathrm{NaCl}$. The troxerutin solution was also prepared in phosphate buffer solution, with the same $\mathrm{pH}$ value. Distilled water was used throughout the experiments.

All the fluorescence spectra were measured using a RF5301PC spectrofluorophotometer (Shimadzu, Japan) equipped with a $150-\mathrm{W}$ Xenon lamp, using a quartz cell of $1.0 \mathrm{~cm}$ path length.

Procedures: A 3-mL human serum solution with a concentration of $1 \mu \mathrm{M} \mathrm{L}^{-1}$ was titrated by successive addition of stock of solution of troxerutin $\left(\mathrm{C}_{\text {troxerutin }}=6 \mu \mathrm{M} / \mathrm{L}\right)$. Titrations were carried out manually by a micro-injector. The fluorescence emissions were recorded with exciting wavelength from 250$290 \mathrm{~nm}$ at the temperature of $299 \mathrm{~K}$.

\section{RESULTS AND DISCUSSION}

Fluorescence spectra of HS excited at $250-290 \mathrm{~nm}$ : The fluorescence spectra of human serum with the exciting wavelength 250-290 $\mathrm{nm}$ are shown in Fig. 3.

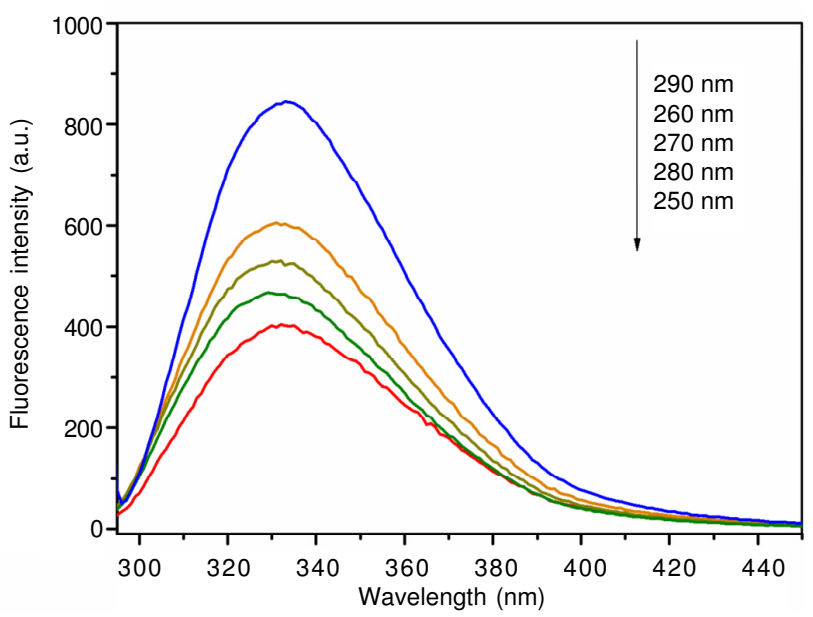

Fig. 3. Fluorescence spectra excited at 250-290 nm
In Fig. 3, there is only one peak at $330 \mathrm{~nm}$ when the sample is excited at wavelength $250-290 \mathrm{~nm}$. The position of the peak $(330 \mathrm{~nm})$ is stable when the wavelength is different.

Fluorescence spectroscopy of the Interaction between human serum and troxerutin: Addition of troxerutin into human serum results in the fluorescence intensity gradually decreases (Fig. 4), indicating the binding of troxerutin to human serum. There is no other peak generating while the concentration of troxerutin is increasing, but the peak has a slight blue shift. And differences of the fluorescence intensity's reducing extent exist when the excited wavelength changes from 250-290 nm with indicating the binding of troxerutin to human serum.

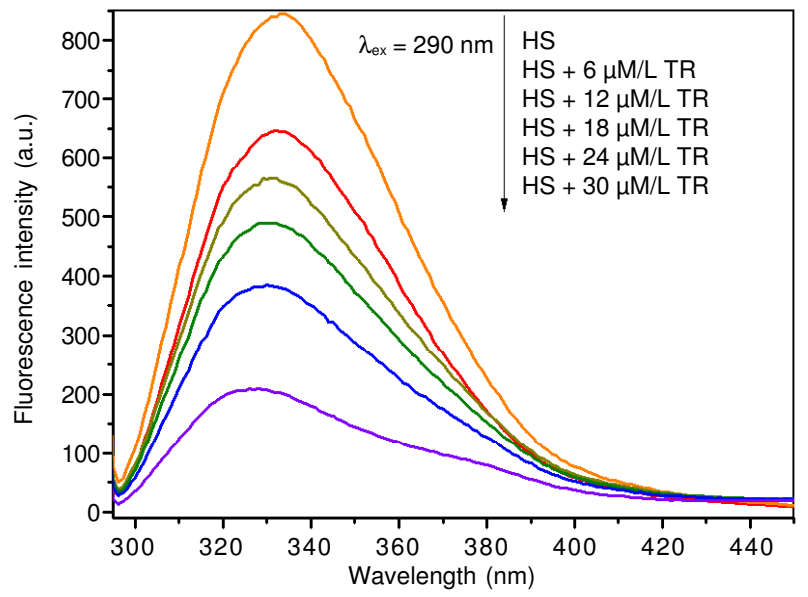

Fig. 4. Fluorescence spectra of the interaction between human serum and troxerutin excitated with $290 \mathrm{~nm}$

In order to clarify the extent of the fluorescence intensity's diminution, the relationship between the concentration of troxerutin and the fluorescence intensity is analyzed in Fig. 5. According to the curves shown in Fig. 5, different slopes occur in the curves when the excited wavelength is in a range of 250-290 $\mathrm{nm}$. In addition, when the excited wavelength is fixed at $290 \mathrm{~nm}$, not only the slope of the curve of the interaction between human serum and troxerutin is the strongest, but also the fluorescence intensity is strongest in all. So the excited wavelength $290 \mathrm{~nm}$ is the best choice for the investigation of interaction between human serum and troxerutin.

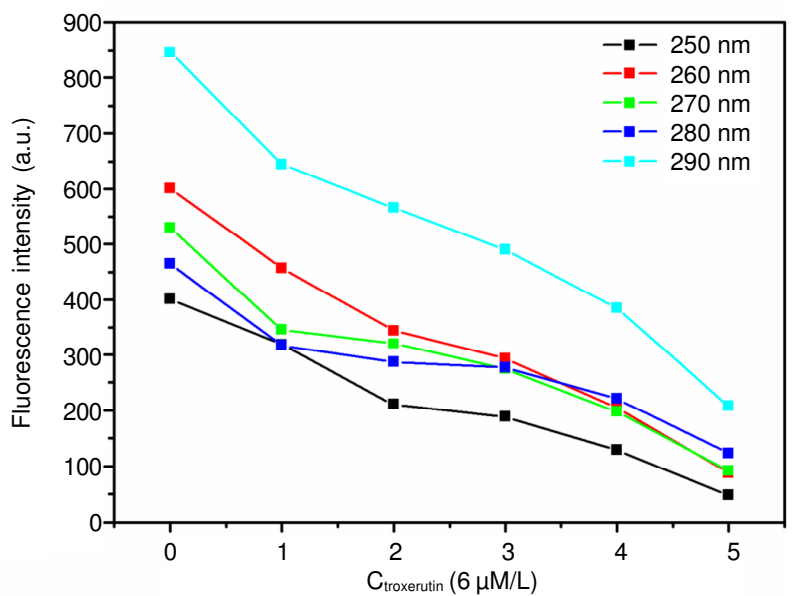

Fig. 5. Relationship between fluorescence intensity of peak and the concentration of troxerutin 
It can be seen from Fig. 3 that human serum's emission wavelength is at $330 \mathrm{~nm}$ and the endogenic fluorescence of troxerutin has no influence on fluorescence spectra of serumtroxerutin's interacton. According to the approximate LambertBeer Law: $\mathrm{I}_{\mathrm{F}}=2.3 \varphi \mathrm{I}_{0} \varepsilon \mathrm{cl}$, where $\mathrm{I}_{\mathrm{F}}$ is the fluorescence intensity, $\varphi$ is emission ratio, $\varepsilon$ is molar extinction coefficient, $\mathrm{c}$ is the sample's concentration, L is optical path length, the fluorescence intensity (IF) is proportional to the sample's concentration $\mathrm{c}^{12}$. As a result, it follows that decrease of the fluorescence intensity illustrate the changes of the concentration of the sample. Perhaps, the decrease of fluorescence intensity due to the decrease of the fluorophore's concentration, which may results from the interaction between human serum and troxerutin. Therefore, it is a clarity for the detection of the interaction between human serum and troxerutin by fluorescence spectroscopy.

Fluorescence spectroscopy study by Gaussion fitting: It is known that human serum is a compound of a variety of ingredient, such as protein, polypeptide. In other words, peaks in the fluorescence spectra may be actually superposition of different fluorescent substance. Generally, a fluorescence spectra of a certain substance is a Gauss curve ${ }^{13}$. Accordingly two Gaussian curves (the centre wavelength are 325 and 350 $\mathrm{nm}$, respectively) fit to the spectra recorded in the experiment by Gaussion fitting (Fig. 6). After the addition of troxerutin, the Gaussian curve with the centre wavelength $350 \mathrm{~nm}$ has a slight red shift gradually. Moreover, the ratio of the intensity of $325 \mathrm{~nm} \mathrm{I}_{325}$ and the intensity of $350 \mathrm{~nm} \mathrm{I}_{350}$ increases when indicating the binding of troxerutin to human serum (Table-1). To the best of our knowledge, the Gaussian curve of the centre wavelength $350 \mathrm{~nm}$ may be the emission of tryptophan and that of the centre wavelength $325 \mathrm{~nm}$ is perhaps the fluorescent line of tyrosine ${ }^{14}$. As a result, the increase of $\mathrm{I}_{325} / \mathrm{I}_{350}$ may demonstrate that tryptophan-troxerutin's interaction is stronger than tyrosine-troxerutin's interaction.

\begin{tabular}{lcccccc}
\multicolumn{7}{c}{ TABLE-1 } \\
\multicolumn{7}{c}{ VALUE OF I $_{325} / \mathrm{I}_{350}$ IN FIG. $6(\mathrm{a}-\mathrm{f})$} \\
\hline & $\mathrm{a}$ & $\mathrm{b}$ & $\mathrm{c}$ & $\mathrm{d}$ & $\mathrm{e}$ & $\mathrm{f}$ \\
\hline $\mathrm{I}_{325}$ & 373 & 333 & 295 & 247 & 142 & 106 \\
$\mathrm{I}_{350}$ & 413 & 302 & 265 & 212 & 97 & 65 \\
$\mathrm{I}_{325} / \mathrm{I}_{350}$ & 0.90 & 1.10 & 1.11 & 1.17 & 1.46 & 1.63 \\
\hline
\end{tabular}

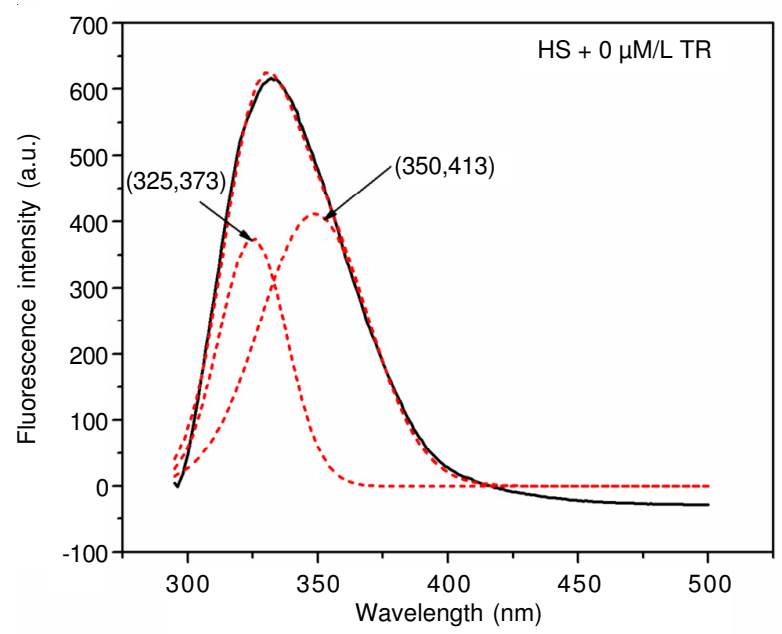

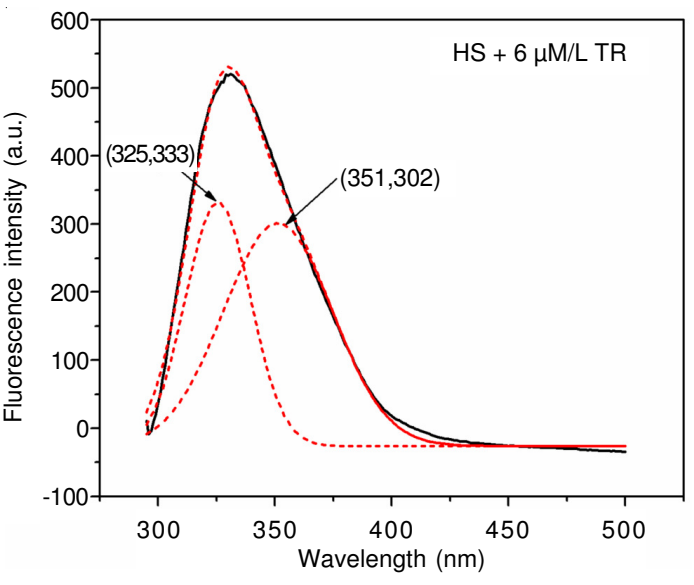
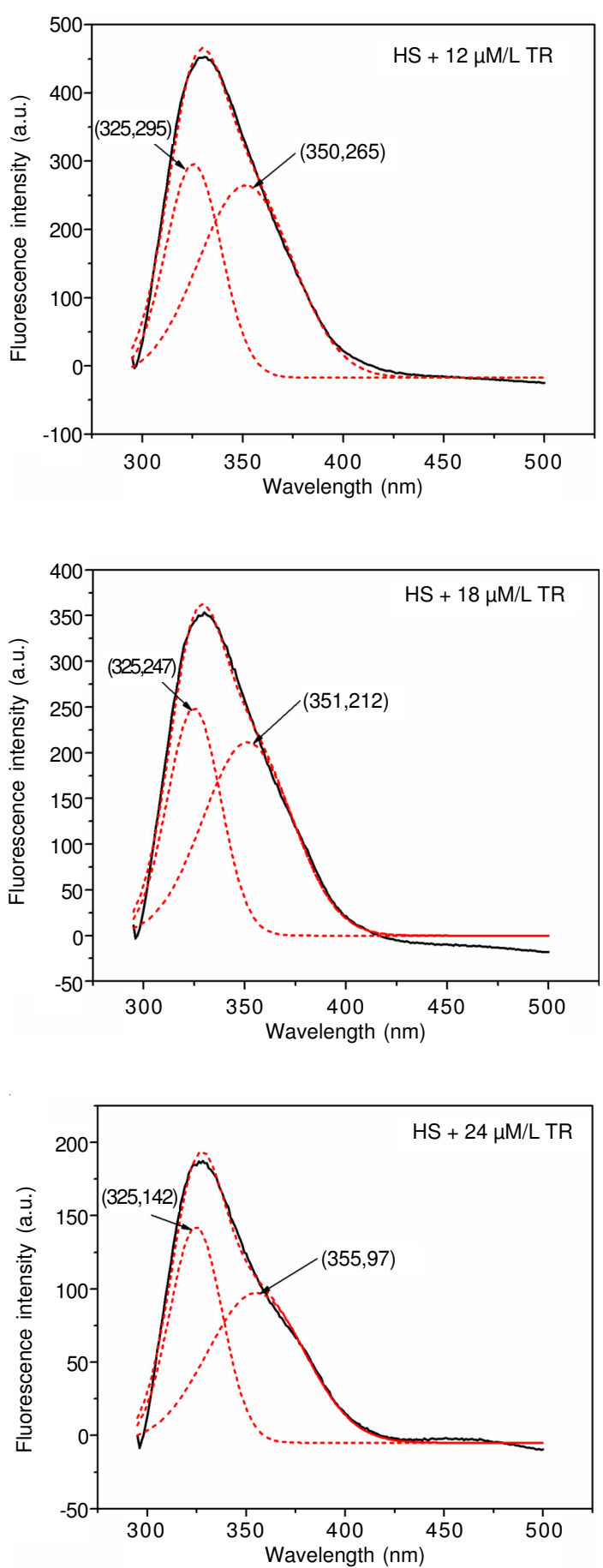


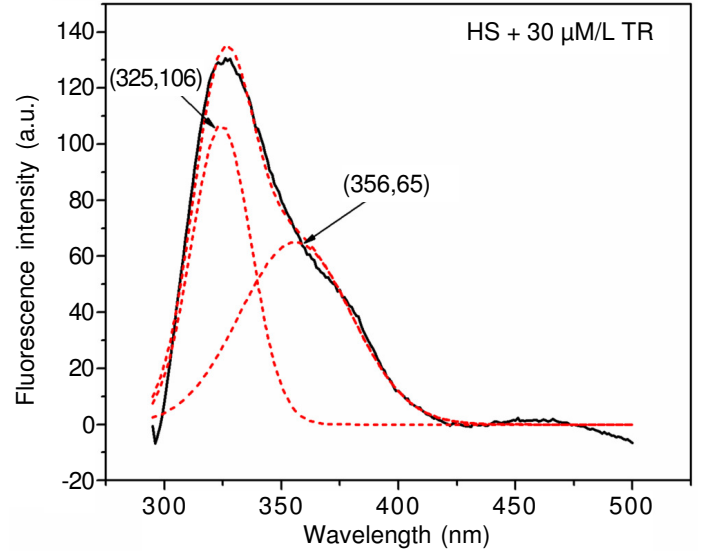

Fig. 6. Guassion fitting of fluorescence spectra excited at $290 \mathrm{~nm}$

\section{Conclusion}

The interaction between human serum and troxerutin was investigated by fluorescence spectroscopy. The result showed that the fluorescence of human serum can be quenched by troxerutin. There is a discrepancy when the sample was excited with varying wavelength (from 220-290 nm). It could be obtained that $290 \mathrm{~nm}$ is most appropriate for the experimental research on serum-troxerutin's interaction. Gaussion fitting curve showed that the centre wavelength was $325 \mathrm{~nm}$, which may be the fluorescent line of tyrosine and the other Gaussion curve perhaps the emission fluorescence spectra of tryptophan.

\section{ACKNOWLEDGEMENTS}

This work is financially supported by International Science and Technology Cooperation Program of Jiangsu
Province of China (No. BZ2010060), Doctoral Fund of Ministry of Education of China (20093218110024) and the Scientific Research Foundation for the Returned Overseas Chinese Scholars (1008-YAK10001). The authors would like to thank Prof. Yonggang Zhu from the Commonwealth Scientific and Industrial Research Organization (CSIRO) in Australia for comments on this manuscript and useful discussions.

\section{REFERENCES}

1. T.H. Wang, Z.M. Zhao, B.Z. Wei and L. Zhang, J. Mol. Struct., 970, 128 (2010).

2. P. Bourassa, I. Hasni and H.A. Tajmir-Riahi, Food Chem., 129, 1148 (2011).

3. K.A. Bashar, K.J. Masood and A.M. Sajid, Colloids Surf. B, 87, 447 (2011).

4. C. Manivannan and R. Renganathan, J. Lumin., 131, 2365 (2011).

5. M. Toprak, B.M. Aydin and M.A.Y. Onganer, J. Lumin., 131, 2286 (2011).

6. S. Radhakrishnan, N. Selvaraj and A. Sambandam, J. Lumin., 131, 2195 (2011).

7. T.H. Wang, Z.M. Zhao, L. Zhang and L. Ji, J. Mol. Struct., 937, 65 (2009).

8. N. Keswani and N. Kishore, J. Chem. Thermodyn., 43, 1406 (2011).

9. I.B. Martha, A.F. Ricardo and S.C. Maria, Biol. Pharm. Bull., 34, 1301 (2011).

10. B.S. Liu, C.L. Xue and J. Wang, Chin. J. Lumin., 31, 285 (2010).

11. K. Carlsson, A. Patwardhan and J.C. Poullain, J. Mal. Vasc., 21, 270 (1996).

12. G.Z. Chen, Fluorescence Analytical Approach, Beijing: Science Press (1990).

13. J. Lu, S.M. Gao and J. Xiong, Laser Technol., 34, 45 (2010).

14. J.R. Lakowicz, Principles of Fluorescence Spectroscopy, Beijing: Science Press, edn. 3 (2008). 